\title{
Radix entomolaris - A case report
}

\author{
Alia Mukhtar', Anamika Borkar ${ }^{2}$, Mohd Sajad ${ }^{3, *}$, Sameena Tabassum ${ }^{4}$ \\ ${ }^{1,3,4}$ Senior Resident, Dept. of Conservative Dentistry and Endodontics, Government Dental College and Hospital, Srinagar, \\ ${ }^{2}$ Professor, Dept. of Conservative Dentistry and Endodontics, Dr. D.Y. Patil Dental College and Hospital, Pune, \\ Maharashtra, India
}

\section{*Corresponding Author: Mohd Sajad}

Email: drmohdsajad1986@gmail.com

\begin{abstract}
Mandibular molars can have an additional root located lingually (the radix entomolaris) or buccally (the radix paramolaris). If present, an awareness and understanding of this unusual root and its root canal morphology can contribute to the successful outcome of root canal treatment. This paper describes case report of mandibular first molar with three roots (one mesial and two distal) and four canals (two in mesial and two in distal root). These case reports show an anatomic variation of internal morphology of the tooth and points out the importance of searching for additional canals.
\end{abstract}

Keywords: Anatomical variations, Endodontic treatment, Mandibular molars, Radix entomolaris, Radix paramolaris.

\section{Introduction}

A clinician should be aware of the internal morphology of permanent teeth and the possible variations which may be encountered. The majority of mandibular first molars are two rooted with one mesial root and one distal root. ${ }^{1}$ The number, location of roots and root canals of these teeth may vary. An additional third root in mandibular molar was first mentioned in the literature by Carabelli, is called the Radix Entomolaris (RE). ${ }^{2}$ In RE, the supernumerary root is located distolingual to the mesial root of the mandibular molars. Similarly an additional root at the mesiobuccal side of the distal root of the mandibular molar is called the Radix Paramolaris (RP). ${ }^{3}$ The prevalence of these three-rooted mandibular first molars appears to be less than $3 \%$ in African populations, not to exceed $4.2 \%$ in Caucasians, to be less than $5 \%$ in Eurasian and Asian populations, and to be higher than $5 \%$ (even up to $40 \%$ ) in populations with Mongolian traits ${ }^{3}$ and $5.97 \%$ in Indian population. ${ }^{4}$ An awareness and understanding of the presence of unusual root canal morphology can thus contribute to the successful outcome of root canal treatment. This report discusses endodontic treatment of three rooted mandibular molars with Radix Entomolaris.

\section{Case}

A 25 year old female patient was reported to the Department of Conservative Dentistry and Endodontics, Dr. D. Y. Patil Dental College and Hospital Pimpri, Pune with a complaint of pain in lower right back tooth since last 5 days. Pain was spontaneous, sharp continuous, aggravates while eating relieved with medication for some time. Intra oral periapical radiograph examination reveals radiolucency involving enamel, dentin and pulp was seen with relation to 46. (Fig. 1) Tooth showed positive response on vitality testing, and was tender on percussion. This radiograph also showed that the tooth had an additional distolingual root. Diagnosis of acute irreversible pulpitis was made and root canal treatment was recommended. Local anaesthesia (inferior alveolar nerve block) was performed. The access cavity was prepared with distolingual extension to provide proper access to distolingual canal. Canal orifices were located, and a radiograph was taken to determine the working length of the canals with two instruments in mesial root and two instrument in the distal root. (Fig. 2) Cleaning and shaping was performed using rotary pro-taper files (Dentsply Maillefer, Swiss made CH-1338 Ballaigues) in crown down manner. Apical preparation was done till size F2 protaper file (master apical file). The canals were irrigated with $3.25 \%$ sodium hypochlorite, $0.2 \%$ chlorhexidine gluconate (Vishal Dentocare Pvt, Ltd India) during instrumentation and finally with normal saline. During preparation, RC HELP (17\% EDTA) was used as a lubricant. The canals were then dried with paper points, master cone selection radiograph was taken,(Fig. 3) and obturated with laterally condensed gutta percha (Dentsply Maillefer, Swiss made $\mathrm{CH}-1338$ Ballaigues) and AH plus sealer(Dentsply DeTreyGmbH, Germany) (Fig. 4).

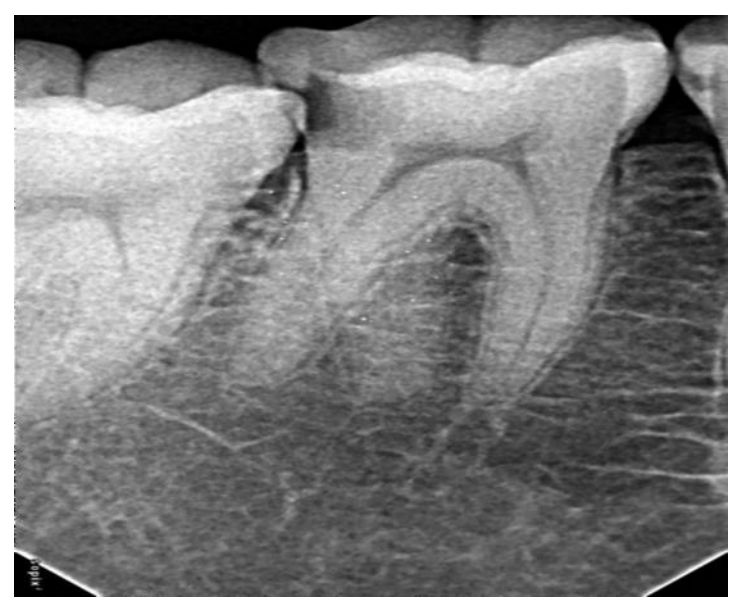

Fig. 1 


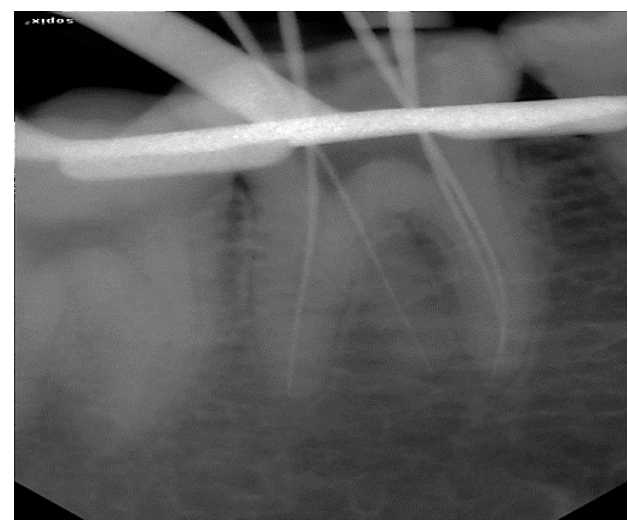

Fig. 2

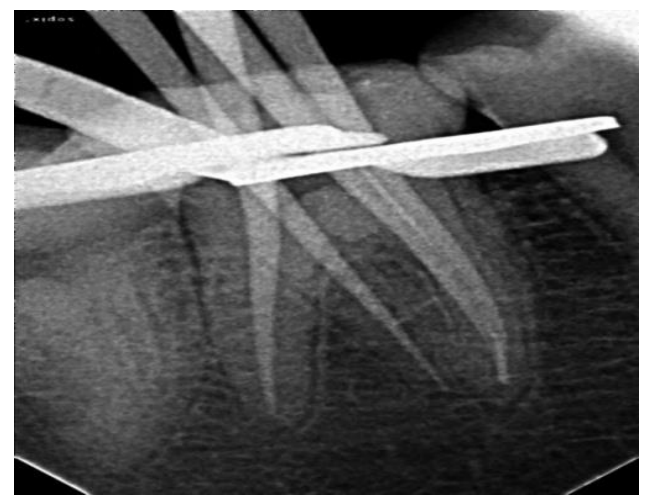

Fig. 3

Fig. 4

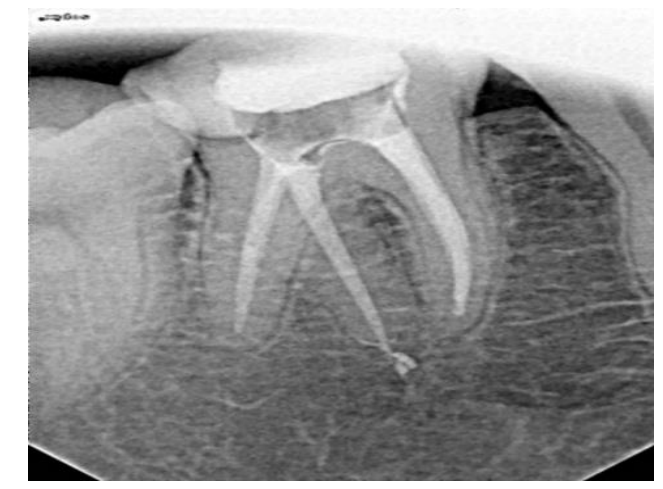

\section{Discussion}

The mandibular first molar can display several anatomical variations. The common morphology that first molars exhibit is two rooted with two mesial and one distal canal. Commonly the mesial root has two root canals, ending in two distinct apical foramina. Sometimes these merge together at the root tip to end in one foramen. The distal root typically has one kidneyshaped root canal, although if the orifice is particularly narrow and round, a second distal canal may be present. ${ }^{5}$ The number of roots may also vary. An additional third root, first mentioned in the literature by Carabelli, is called the Radix Entomolaris (RE). This supernumerary root is located distolingually in mandibular molars. RE was found on the first, second and third mandibular molars, occurring least frequently on the second molar. Some studies reported a bilateral occurrence of the RE from $50 \%$ to $67 \%$. Similarly additional root in the mesiobuccal side is called the radix paramolaris (RP). ${ }^{2}$ The prevalence of these three rooted mandibular first molars appears to be Less than 3\% in African populations, $4.2 \%$ in Caucasians, Less than $5 \%$ in Eurasian and Asian populations Higher than 5\% (even up to $40 \%$ ) in populations with Mongolian traits. ${ }^{2}$ Bolk reported the occurrence of a buccally located additional root: the RP. This macrostructure is very rare and occurs less frequently than the RE. The prevalence of RP, as observed by Visser, was found to be $0 \%$ for the first mandibular molar, $0.5 \%$ for the second and $2 \%$ for the third molar. ${ }^{6}$

\section{Etiology}

The etiology behind the formation of RE is still unclear. In dysmorphic, supernumerary roots, its formation could be related to external factors during odontogenesis, or to penetrance of an atavistic gene or polygenetic system. ${ }^{7}$

\section{Morphology of the Radix Entomolaris}

The RE is located distolingually, with its coronal third completely or partially fixed to the distal root. The dimensions of the RE can vary from a short conical extension to a 'mature' root with normal length and root canal. In most cases the pulpal extension is radiographically visible. In general, the RE is smaller than the distobuccal and mesial roots and can be separate from, or partially fused with, the other roots. ${ }^{2}$

Classification by Carlsen and Alexandersen describes four different types of RE according to the location of the cervical part of the RE: Types A, B, C and AC. Types A and B refer to a distally located cervical part of the RE with two normal and one normal distal root components, respectively. Type $\mathrm{C}$ refers to a mesially located cervical part, while Type AC refers to a central location, between the distal and mesial root components. This classification allows for the identification of separate and nonseparate RE. According to the classification of De Moor et al. based on the curvature of the separate RE variants in buccolingual orientation, three types can be identified. Type I refers to a straight root/root canal, Type II refers to an initially curved entrance which continues as a straight root/root canal. Type III refers to an initial curve in the coronal third of the root canal and a second curve beginning in the middle and continuing to the apical third. $^{2}$

\section{Clinical Approach}

The presence of an RE or an RP has clinical implications in endodontic treatment. An accurate diagnosis of these supernumerary roots can avoid complications or a 'missed canal' during root canal treatment. As the (separate) RE is mostly situated in the 
same buccolingual plane as the distobuccal root, a superimposition of both roots can appear on the preoperative radiograph. A thorough inspection of the preoperative radiograph and interpretation of particular marks or characteristics, such as an unclear view or outline of the distal root contour or the root canal, can indicate the presence of a 'hidden' RE. To reveal the RE, a second radiograph should be taken from a more mesial or distal angle (30 degrees). ${ }^{2}$ This way an accurate diagnosis can be made in the majority of cases. Apart from a radiographical diagnosis, clinical inspection of the tooth crown and analysis of the cervical morphology of the roots by means of periodontal probing can facilitate identification of an additional root. An extra cusp (tuberculum paramolare) or more prominent occlusal distal or distolingual lobe, in combination with a cervical prominence or convexity, can indicate the presence of an additional root. The location of the orifice of the root canal of an RE has implications for the opening cavity. The orifice of the RE is located disto- to mesiolingually from the main canal or canals in the distal root. An extension of the triangular opening cavity to the (disto) lingual results in a more rectangular or trapezoidal outline form. If the RE canal entrance is not clearly visible after removal of the pulp chamber roof, a more thorough inspection of the pulp chamber floor and wall, especially in the distolingual region, is necessary. Visual aids such as a loupe, intra-oral camera or dental microscope can, in this respect, be useful. ${ }^{8}$ A dark line on the pulp chamber floor can indicate the precise location of the RE canal orifice. The distal and lingual pulp chamber wall can be explored with an angled probe to reveal overlying dentin or pulp roof remnants masking the root canal entrance. The calcification, which is often situated above the orifice of the RE, has to be removed for a better view and access to the RE. An initial relocation of the orifice to the lingual is indicated to achieve straight-line access. However, to avoid perforation or stripping in the coronal third of a severe curved root, care should be taken not to remove an excessive amount of dentin on the lingual side of the cavity and orifice of the RE. severe root inclination or canal curvature, particularly in the apical third of the root, can cause shaping aberrations such as straightening of the root canal or a ledge, with root canal transportation and loss of working length resulting. The use of flexible nickel-titanium rotary files allows a more centered preparation shape with restricted enlargement of the coronal canal third and orifice relocation. Complications such as instrument separation do occur, and are more likely to happen in an RE with severe curvature or narrow root canals. Therefore, after relocation and enlargement of the orifice of the RE, initial root canal exploration with small files (size 10 or less) together with radio graphical root canal length and curvature determination, and the creation of a glide path before preparation, are step-by-step actions that should be taken to avoid procedural errors. ${ }^{2}$

\section{Conclusion}

Clinicians should be aware of these unusual root morphologies in the mandibular first molars. The initial diagnosis of a radix entomolaris or paramolaris before root canal treatment is important to facilitate the endodontic procedure, and to avoid 'missed' canals. Preoperative periapical radiographs exposed at two different horizontal angles are required to identify these additional roots. Knowledge of the location of the additional root and its root canal orifice will result in a modified opening cavity with extension to the distolingual. The morphological variations of the RE in terms of root inclination and root canal curvature demand a careful and adapted clinical approach to avoid or overcome procedural errors during endodontic therapy.

\section{References}

1. Vertucci FJ. Root canal anatomy of the human permanent teeth. Oral Surgery, Oral Medicine, Oral Pathology. 1984;58(5):589- 99.

2. Calberson FL, De Moor RJ, Deroose CA. The radix entomolaris and paramolaris: clinical approach in endodontics. Journal of Endodontics. 2007;33(1):58-63.

3. De Moor R, Deroose C, Calberson F. The radix entomolaris in mandibular first molars: an endodontic challenge. International Endodontic Journal. 2004;37(11):789-99.

4. Garg AK, Tewari RK, Kumar A, Hashmi SH, Agrawal N, Mishra SK. Prevalence of three rooted mandibular first Molars among the Indian Population. Journal of Endodontics. 2010;36(8):1302-5.

5. Segura-Egea JJ, Jiménez-Pinzón A, Rios-Santos JV. Endodontic therapy in a 3-rooted mandibular first molar: importance of a thorough radiographic examination. $J$ Can Dent Assoc. 2002;68(9):541-4.

6. Bolk L. The importance of endodontic in maxillary and mandibular molar canals. J Can Dent Assoc. 1994;60:527-32.

7. Vimala N, Firake N. Know your mandibular first molar: a case report. Sci J. 2009; Vol. III.

8. Feix LM, Boijink D, Ferreira R, Wagner RH, Barletta FB. Microscópio operatório na Endodontia: magnificação visual e luminosidade. RSBO. 2010;7(3):340-8. 